\title{
The diagnostic potential of microneedles in infectious diseases
}

\author{
Rachael V. Dixon ${ }^{1,2}$, Wing Man Lau ${ }^{1,2}$, S. Moein Moghimi ${ }^{1,2}$, Keng Wooi $\mathrm{Ng}^{1,2, *}$ \\ ${ }^{1}$ School of Pharmacy, and ${ }^{2}$ Translational and Clinical Research Institute, \\ Faculty of Medical Sciences, Newcastle University, Newcastle upon Tyne, UK.
}

\section{Graphical Abstract}
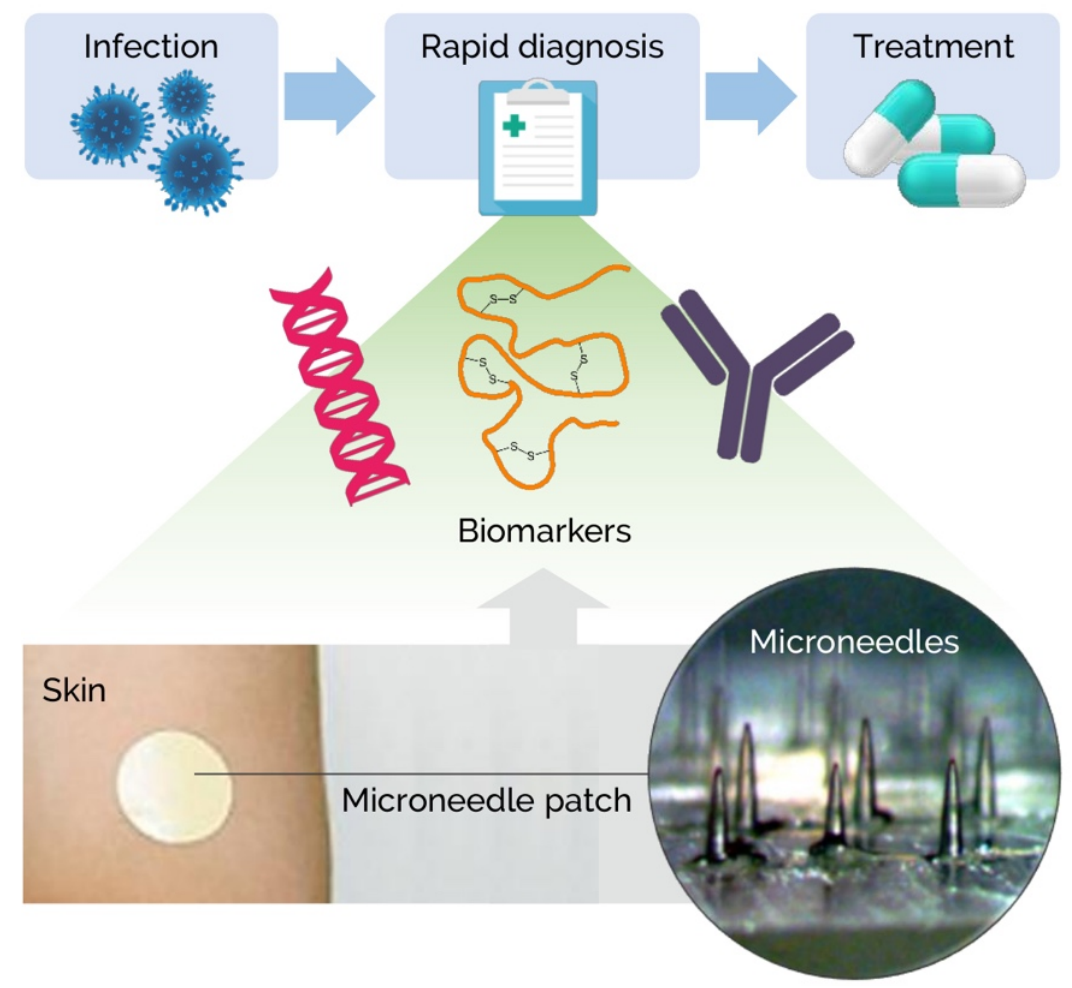

Point-of-care microneedle diagnostics

\section{Abstract}

The coronavirus disease 2019 (COVID-19) pandemic has taught us much about our weaknesses in the management of infectious disease outbreaks. A key lesson has been the need for more effective pointof-care diagnostic tools that produce not only rapid and reliable results but also facilitate decentralised testing to avoid overwhelming central test facilities when demand peaks in an outbreak. Microneedle devices can be inserted painlessly into the skin to detect biomolecules in the epidermal and dermal layers. They have been used to identify biomarkers in both the interstitial fluid and capillary blood. Importantly, they are amenable to self-administration. In this article, we provide an overview of existing microneedle-based diagnostic technologies and discuss how they may be built upon to provide effective diagnostic tools for infectious diseases.

\section{Keywords}

Infection, microneedles, biosensor, antigen capture, skin, interstitial fluid, biomarker, point-of-care diagnostics, infectious diseases

*Corresponding author: keng.ng@newcastle.ac.uk; tel.: +44 (0) 1912082343 


\section{Purpose \& Rationale}

In late January 2020, during the peak of the lunar New Year travel season, the Chinese government took the extraordinary measure of locking down entire cities to contain a new and highly contagious viral respiratory disease. The media reports at the time provided the first indications of the unprecedented public health challenges posed by the coronavirus disease 2019 (COVID-19) outbreak. As hospitals experienced a sudden influx of potentially infected patients, many patients waited to be seen in overcrowded hospital lounges.

By March 11, 2020, the infection had spread rapidly across multiple continents, prompting the World Health Organization (WHO) to declare a pandemic. Since then, the scenes of overstretched public health systems that are struggling to meet the sudden spike in demand for COVID-19 diagnoses have been replicated in many countries, even among the richest economies. The COVID-19 outbreak has upended the common perception that infectious diseases are a problem of the developing world and exposed many deficiencies in current diagnostic strategies for infectious diseases.

Here, we discuss the lessons to be learned from this episode and how emerging microneedle diagnostic technologies could potentially help avert similar challenges in the future.

\section{Introduction \& Discussion}

In recent decades, several infectious disease outbreaks have caused major disruptions in both the developed and developing regions of the world. For example, epidemics of dengue fever, the Ebola virus disease, and the Zika virus diseases ravaged Africa, Asia, and South America between 2014 and 2016. The world has also witnessed successive outbreaks of respiratory viral infections, including various strains of the influenza virus, as well as coronaviruses that caused the severe acute respiratory syndrome (SARS, 2002), the Middle East respiratory syndrome (MERS, 2012), and the COVID-19 (2019) pandemic that still grips the world at the time of this writing. In 2016, mortality rates by infectious diseases accounted for approximately 8.5 million deaths globally, more than half of which were recorded in lowincome countries [1].
Infectious disease outbreaks are not new. The 1918 Spanish flu remains one of the most devastating outbreaks in modern history [2], and infectious disease outbreaks have been linked to the collapse of entire civilisations [3]. While modern-day healthcare systems across the world have adapted to manage gradual developments of public health threats, they are generally ill-equipped to respond to sudden outbreaks of infectious diseases. For the past few years, infectious disease pandemics and a lack of access to affordable diagnostic tools have featured every year on WHO's annual list of predicted health challenges and threats to global health [4]-[6]. However, despite ample warnings, the world continued to be underprepared for an infectious disease pandemic.

Then, in 2019, the threat materialised sooner than expected. COVID-19 was first reported in Wuhan, China, in December 2019 and rapidly spread throughout the world. As of May 27, 2020, the global death toll from COVID-19 stood at over 350,000 , with approximately three-quarters of the deaths in high-income countries, including the United States, the United Kingdom, Italy, Spain, France, and Belgium [7], [8]. However, the number of tests performed per capita remained generally below $6 \%$ [8]. Amid calls for more tests to be done [9], [10], governments admitted that testing capacity was limited [11], [12].

These examples illustrate that it is not just in developing and underdeveloped countries that infectious disease diagnosis can be a challenge. Developed countries, too, face challenges due to centralised - albeit state-of-the-art - test facilities and limited resources that can be overwhelmed in the case of a severe infectious disease outbreak.

\section{What do we need for effective diagnosis in an infectious disease outbreak?}

Effective diagnostics should be at the heart of our global strategy for tackling infectious diseases so that infected individuals can be quickly identified and treated, to effectively monitor and control disease transmission. Point-of-care diagnostics is medical testing administered near or at the point of patient care. Point-of-care diagnostic tests for infectious disease should fulfil the ASSURED criteria: be affordable, sensitive, specific, $u$ ser friendly, rapid and robust, equipment-free, and deliverable to end-users 
[13]. Some current point-of-care tests already meet these criteria to various extents [14], but some significant challenges remain.

Current diagnostic protocols for most infectious diseases hinge on the collection of bodily fluids for laboratory tests that require skilled personnel and expensive equipment. Many of these samples require "cleaning up" to remove cellular debris and assay inhibitors before the target marker/molecule can be processed. The prepared sample is then analysed using state-ofthe-art techniques such as the polymerase chain reaction (PCR) or immunoassays to detect nucleic acids, pathogenic antigens, or host antibodies. These complex procedures restrict the use of such tests to central test facilities like hospitals and specialist laboratories, requiring the public to travel to clinics and hospitals to be tested. Herein lie several problems. First, centralised test facilities limit the accessibility of the test. Second, the diagnostic procedure necessitates close contact between potentially infected individuals and healthcare professionals, which increases the risk of disease transmission to key personnel. Third, in the case of an infectious disease outbreak, requiring potentially infected individuals to travel to test facilities defeats the objective of any quarantine measures that may be in place to help contain the disease. We have witnessed these problems play out in the COVID-19 pandemic where strict travel restrictions were imposed in many regions of the world, and internal lockdown measures were implemented to encourage the public to stay at home. Meanwhile, the global demand for critical personal protective equipment and accurate diagnostic tests skyrocketed while supply chains were disrupted, leading to acute shortages.

To overcome these limitations, more testing tools that are suitable for home use need to be developed. In addition to meeting the ASSURED criteria above, such tests should ideally allow self-diagnosis by being self-administrable and self-analysable. As an example, the home pregnancy test provides an ideal platform to which infectious disease diagnostics can aspire. The ability to self-diagnose infections rapidly and reliably will not only accelerate diagnosis and treatment but also alleviate the strain on central test laboratories and health workers. Another advantage is that the public would not need to travel to overcrowded centralised healthcare facilities for testing, risking that they might infect others or be infected in the process.

However, such a test kit will still rely on a functioning supply chain and distribution network to work effectively. While such a diagnostic kit may seem far-fetched for infectious disease outbreaks, microneedle-based diagnostic platforms promise the technological leap necessary to realise this advancement.

\section{Microneedle diagnostic platforms}

Typically presented as a skin patch, microneedles are arranged into a grid array with solid or hollow needle shafts $<1 \mathrm{~mm}$ long and up to a few hundred micrometres wide, projecting perpendicularly from the patch surface (Figure 1). When applied on the skin, the microneedles create micropores in the superficial skin tissue. The application of microneedle patches has been shown to be painless and better accepted by users compared with conventional hypodermic needles [15]-[18]. Microneedles are short enough to penetrate only the superficial skin layers, avoiding nerve endings and major blood vessels, while still accessing analyte-rich interstitial fluid (ISF) or capillary blood. ISF is an attractive target because it contains many of the same constituents as blood plasma and suction blister fluid at clinically relevant concentrations [19]-[21]. Encouragingly, it has already been demonstrated in the context of drug delivery that microneedle patches are self-administrable [22]-[24], which paves the way for self-administration of microneedle-based diagnostics.

Microneedle diagnostic platforms have a long history of development for applications such as glucose monitoring, but infectious disease detection is an emerging application, and there are reasons to be optimistic about this potential use. Fueled by advances in the underpinning technologies, microneedle diagnostic technologies have recently witnessed remarkable innovations. For example, until recent years, PCR was performed almost exclusively in central facilities with specialist personnel and thermocycling equipment [25]-[27]. The emergence of isothermal PCR has allowed nucleic acids to be amplified close to body temperature [27]-[31]. 

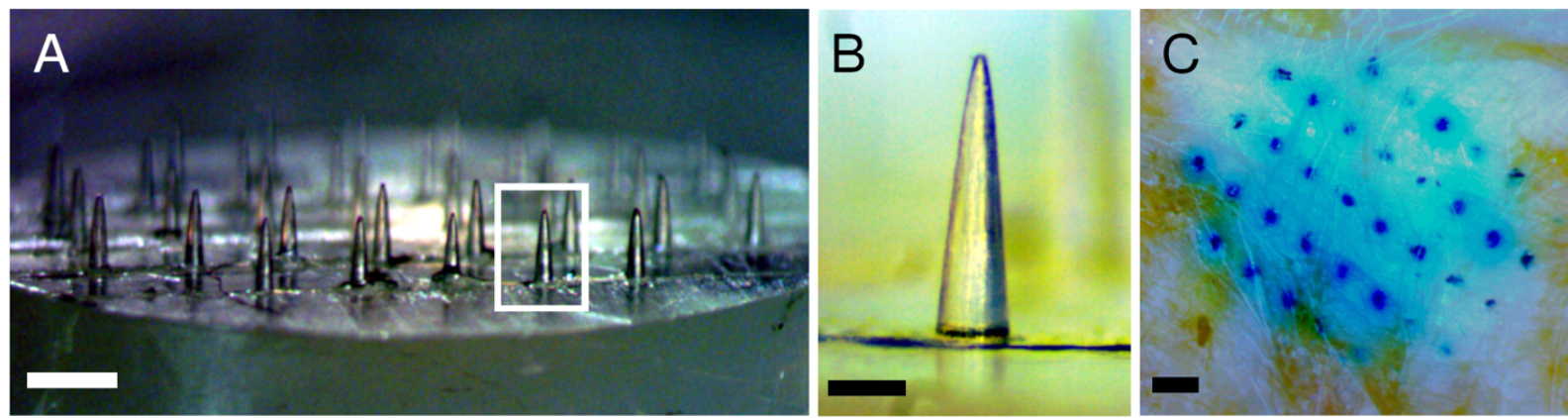

Figure 1: (A) An exemplar microneedle array made of polylactic acid. The boxed region in (A) is enlarged in (B) to show a single microneedle. (C) Micropores created by a microneedle array in the skin, highlighted by a blue dye (methylene blue) for ease of visualisation. Scale bars: $(A, C) 1 \mathrm{~mm}$; (B) $250 \mu \mathrm{m}$.

This has, in turn, inspired the development of a wearable microneedle patch to detect the Epstein-Barr virus which amplifies the target viral circulating-free deoxyribonucleic acid (DNA) in situ by isothermal PCR between $32^{\circ} \mathrm{C}$ and $37^{\circ} \mathrm{C}$ [32]. This practice paves the way for using microneedle diagnostic technologies to detect a host of other infectious diseases with known circulating-free DNA targets, including malaria and tuberculosis [33], [34]. Recent advances in other areas closely related to skin bi-

(A) Biofluid extraction Hollow microneedles

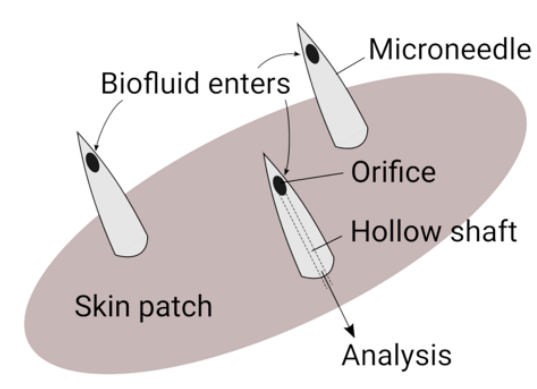

(C) Analyte capture

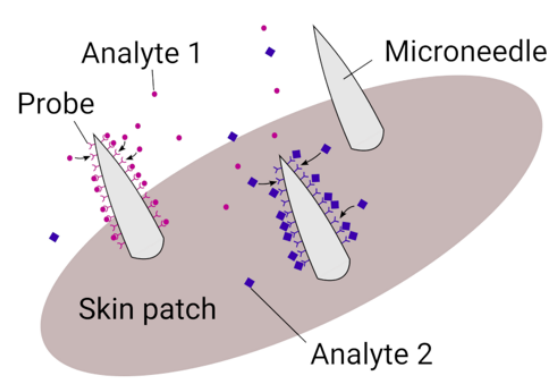

osensing and bioanalysis, such as nanotechnology and 3D printing, will likely further enhance microneedle-based diagnostics [35].

To understand how microneedles can be leveraged for infectious disease diagnosis, we need to dissect how they work. Broadly speaking, current microneedle diagnostic systems operate in one of the following ways: (1) they extract biofluids or target analytes from the skin for in vitro testing or (2) they perform in situ biosensing of target analytes in the skin (Figure 2).

\section{(B) Biofluid extraction Solid microneedles}

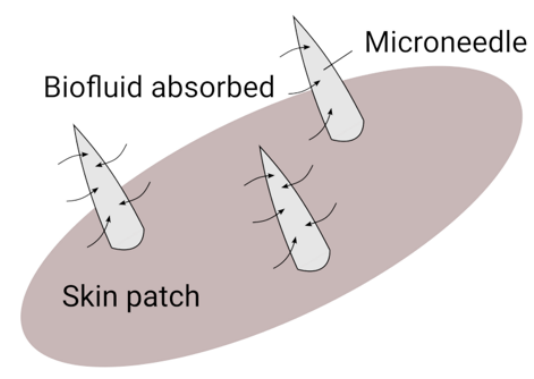

\section{(D) Electrochemical biosensing}

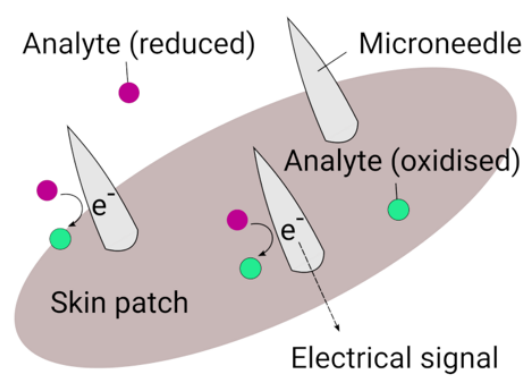

Figure 2: Common modes of action of current microneedle diagnostic platforms. (A, B) Biofluid extraction can be achieved using hollow or solid microneedles. (C) Specific target analyte capture can be achieved using immobilised probes on microneedles. A multiplex system designed to detect analytes 1 and 2 simultaneously is shown. (D) Electrochemical microneedle biosensors convert a redox reaction into an electric signal. 
The mode of action depends largely on the target analyte, which influences the choice of the downstream analytical procedure. These devices may be coupled with conventional analytical equipment, or sophisticated lab-on-a-chip analytical systems may be integrated into the microneedle diagnostic platforms. In either case, the hallmark of these microneedle-based diagnostic technologies is their ability to easily access skin biofluids (e.g., ISF and capillary blood) with minimal invasiveness.

\section{Microneedles for biofluid extraction}

Biofluids can be extracted from the skin using hollow or solid microneedles, the former having been particularly well demonstrated in the context of microneedle-based glucose biosensors. Hollow microneedles have a hollow needle shaft and a surface orifice. Biofluid uptake through the orifice into the hollow microneedle shaft could occur by capillary action, or biofluid flow could be induced through a microfluidic system, usually by applying negative pressure [37]-[42]. This technique has been used to extract dermal ISF using hollow silicon microneedles for on-chip detection (see "Microneedles for in situ biosensing," below), as well as offchip nucleic acid profiling to identify potential disease biomarkers, in conjunction with conventional sample processing techniques and PCR-based analysis [43]. One study reported an ISF uptake rate of $1 \mu \mathrm{L} / \mathrm{s}$ [39].

Solid microneedles, on the other hand, do not have a surface orifice or a hollow shaft. The application of solid microneedles encourages ISF to migrate to the skin's surface. ISF can then be absorbed into a sheet of paper for subsequent biomarker analysis [44], [45]. Alternatively, hydrogel-forming microneedles swell upon insertion into the dermal interstitium and absorb ISF. Downstream processing of the hydrogel microneedle array by centrifugation liberates the analytes of interest, which can then be quantified using conventional techniques such as high-performance liquid chromatography [46][48]. Porous microneedle arrays contain interconnected pores in the body of the microneedles, which allow them to absorb skin biofluids by capillary action. The biofluids can then be recovered from the microneedles by centrifugation [49], [50]. Biofluid flow can also be actively induced. In one study using stainless steel solid microneedles and a suction pump, $100 \mu \mathrm{L}$ of capillary blood was extracted through microfluidic channels into a collection chamber in 3 minutes [51].

Biofluid extraction using microneedles is advantageous primarily because it is minimally invasive and quick. However, a major challenge of this approach is the limited volume of ISF or capillary blood extracted, which is typically in the nanoliter-to-microliter range. Larger sample volumes are usually required for current lab-based analytical techniques or commercially available testing kits [52]-[57]. While it is possible to extend the microneedle length to sample larger volumes directly from larger blood vessels, doing so inevitably increases the technique's invasiveness and complexity, diminishing the advantages of being minimally invasive and self-administrable. Also, hollow microneedles can become clogged with tissue debris, although some advances have been made to resolve this issue [58]. In comparison, solid microneedles are simpler in design and hence are likely to be easier and cheaper to manufacture at scale. However, the downstream processes to liberate the analyte of interest from solid microneedle systems typically dilute the sample, thus reducing the analyte concentration. Coupling such microneedle biofluid extraction devices to current analytical equipment may prove challenging for lowabundance analytes.

Moreover, the extracted samples still require purification to isolate the analyte of interest prior to analysis. This may be advantageous as it could be possible to perform the analysis using existing analytical facilities, so the barrier to adoption may be low. Nonetheless, it will rely on specialist personnel and central analytical facilities, which may prove to be a bottleneck in achieving the rapid diagnosis desired. To decentralise the analytical step, a lab-on-achip microneedle diagnostic platform with integrated analytics is desirable. On-chip detection has already been demonstrated for metabolites, including glucose and cholesterol using microneedle devices with integrated colourimetric assays that promise to be simple, quick, and economical. For example, biofluids have been absorbed into paper strips containing reagents that undergo a measurable color change in the presence of the target analyte [59], [60]. This style of point-of-care test can easily be multiplexed by incorporating multiple test strips with 
different detection reagents. It may be possible to adapt this strategy for infectious disease diagnosis, for example, as an on-chip colourimetric immunoassay for detecting pathogenic antigens.

\section{Microneedles for analyte capture}

Some microneedle devices can extract only specific target analytes from the skin without removing any perceivable amount of biofluid. This technique is advantageous over biofluid extraction as it eliminates the need for sample purification. Target analytes with structural motifs amenable to molecular recognition, such as proteins and nucleic acids, may be extracted using this technique.

One embodiment of this technology uses antigen-specific immunocapture. Capture antibodies immobilised on to the surface of solid microneedles have successfully captured pathogenic antigens, host antibodies, and cytokines from the skin at concentrations comparable to the assay limits of conventional immunoassays [61]-[66]. Target capture at detectable levels in under 1 minute has been demonstrated [67]. By immobilising different capture antibodies on to different microneedle surfaces on a given array, multiplex detection (i.e., simultaneous detection of multiple analytes) and internal assay controls can be incorporated [65]. Post-capture antigen confirmation and quantification can be accomplished using conventional techniques such as colourimetry and fluorimetry. The development of more facile and portable analytical techniques, such as paper-based visual analysis [65], [66], may facilitate self-analysis by lay users. Longer application times of an immunocapture microneedle patch may facilitate analyte detection at lower concentrations but may prolong the test duration. A delicate balance will likely need to be struck between these considerations.

Similarly, peptide nucleic acids immobilised on the surface of hydrogel microneedles have successfully captured target micro-ribonucleic acid (microRNA) sequences in skin ISF by hybridisation [68]. The captured microRNA was detected and quantified "on-patch" using fluorescently tagged DNA probes, or "off-patch" by liberating the hybridised microRNA complexes via a photocleavable linker molecule in the peptide nucleic acid probe. This method of nucleic acid extraction and purification promises significant time and cost savings over conventional methods.

These techniques rely on a high-quality surface for probe (i.e., capture antibody or peptide nucleic acid probe) immobilisation, as well as strong and specific intermolecular interactions between the probe and target analyte. Therefore, the microneedle fabrication technique should ideally promote high-density probe immobilisation and optimal probe orientation and minimise non-specific binding while ensuring that the microneedle surface is biocompatible. Various immobilisation strategies are available, and their success will depend largely on the properties of the initial deposition surface [67], [69]. Furthermore, increasing the number of microneedles per array will increase the surface area for probe deposition, thus increasing device sensitivity and its multiplexing potential.

\section{Microneedles for in situ biosensing}

Electrochemical microneedle biosensors can detect redox reactions induced by the analyte of interest in situ and produce electrical measurements proportional to the analyte concentration. These technologies can comprise hollow or solid microneedles and have been explored mainly for the detection of small molecules, including glucose, lactate, uric acid, nitric oxide, ascorbic acid, cholesterol, and levodopa [70][74]. Hollow microneedles are typically used to extract and channel biofluids to the electrodes of the electrochemical cell, while solid microneedles usually serve as the electrodes themselves.

The main advantage of in situ biosensing is the real-time or near-real-time analyte detection, without the need for sample processing or storage. However, electrochemical microneedle biosensors demand that the analyte be either electroactive or coupled to a suitable intermediary or transducer system capable of converting a non-electrical event into an electrical signal. For example, the vast majority of glucose biosensors do not detect glucose directly but incorporate glucose oxidase as an enzymatic intermediary. The lack of either option in the analyte is likely to be the key factor limiting the application of these microneedle biosensors.

Analyte detection does not have to be electrochemical in nature, however. For example, 
Smart and Subramanian [74] described a glucose biosensor based on glucose dehydrogenase-formazan chemistry, where glucose concentration was measured by optical absorbance. Although the hollow "microneedles" used in that study were $2 \mathrm{~mm}$ long and so do not fit our definition above, it was nonetheless an excellent demonstration of on-chip analyte detection using a non-electrochemical method.
The literature on in situ biosensors for infectious diseases is scarce. However, a hollow microneedle device was developed to extract ISF from the skin and transfer it into a chamber containing either immobilised anti-CD4 $\mathrm{T}+$ cell antibodies (to detect human immunodeficiency virus or HIV) or antibodies against Ebola capsid glycoproteins (to detect Ebola virus). The cell/ligand binding events were measured by electrical impedance [75]-[77].

\section{Conclusions}

The Global Preparedness Monitoring Board has recently highlighted two pitfalls in the global health emergency response strategy in relation to disease diagnostics: first, national capacities for diagnostics research and development are underdeveloped, and second, diagnostics has received fewer investments than other areas, such as a vaccine, basic research, and therapeutics [78]. Current testing kits for infectious diseases can be expensive, require trained medical personnel, and rely on limited medical infrastructure that is typically centralised. Due to resource limitations, these characteristics could hinder disease control efforts in an infectious disease outbreak.

Decentralised, self-administrable, and self-diagnosing point-of-care tests can help alleviate these resource pressures. Microneedle diagnostic technologies, being minimally invasive, self-administrable, portable, cost-effective, and mass-producible, offer an attractive approach to rapid, decentralised pointof-care infectious disease diagnosis. This is a nascent area of research, so development and manufacturing costs are difficult to predict. The vast majority of microneedle devices are fabricated from materials such as silicon, polymers, and metals using already well-established low-cost techniques including micromolding, machine milling, injection molding, 3D printing, and micro-electromechanical systems manufacture [79], [80], most of which lend themselves to large-scale production. The development and manufacturing costs are likely to be offset by a reduction in the professional labour time, which is the primary cost associated with point-of-care testing [81], [82].

Significant technical challenges also remain especially in terms of method validation and miniaturisation of the detection backend, as well as regulatory and quality control during mass manufacture [83], despite the optimism offered by some examples of successful integration of on-chip analytical systems into microneedle platforms for other medical conditions (discussed above). Overcoming these technical challenges requires a concerted effort of research scientists, industrialists, and policymakers. More resources need to be invested to spur the development of robust point-of-care devices, building on the strengths of current microneedle diagnostic technologies. Intensified research and development will mature the technologies needed to mass-produce microneedle diagnostic devices, which will further reduce the cost and make microneedle-based diagnostics more accessible, even in lower-income regions.

\section{Conflict of Interests}

The authors declare no conflicts of interest. For a signed statement, please contact the journal office: editor@precisionnanomedicine.com

Quote this article as: Dixon RV, Lau WM, Moghimi SM, Ng KW, The diagnostic potential of microneedles in infectious diseases, Precis. Nanomed. 2020;3(3):629-640, https://doi.org/10.33218/001c.13658

\section{References}

[1] World Health Organization, 'The top 10 causes of death', May 24, 2018. https://www.who.int/news-room/fact-sheets/detail/the-top-10-causes-of-death (accessed March 31, 2020).

[2] M. Honigsbaum, 'Spanish influenza redux: revisiting the mother of all pandemics', Lancet, vol. 391, no. 10139, Art. no. 10139, Jun. 2018, doi: 10.1016/S0140-6736(18)31360-6. 
[3] E. Callaway, 'Collapse of Aztec society linked to catastrophic salmonella outbreak', Nature, vol. 542, no. 7642, Art. no. 7642, Feb. 2017, doi: 10.1038/nature.2017.21485.

[4] World Health Organization, 'Ten health issues WHO will tackle this year', April 02, 2020. https://www.who.int/news-room/feature-stories/ten-threats-to-global-health-in-2019 (accessed April $02,2020)$.

[5] World Health Organization, 'Urgent health challenges for the next decade', November 13, 2020. https://www.who.int/news-room/photo-story/photo-story-detail/urgent-health-challenges-forthe-next-decade (accessed April 02, 2020).

[6] World Health Organization, ' 10 threats to global health in 2018', February 15, 2019. https://medium.com/@who/10-threats-to-global-health-in-2018-232daf0bbef3 (accessed April 02, 2020).

[7] European Centre for Disease Prevention and Control, 'COVID-19 situation update worldwide, as of May 27 2020', European Centre for Disease Prevention and Control, May 27, 2020. https://www.ecdc.europa.eu/en/geographical-distribution-2019-ncov-cases (accessed May 28, 2020).

[8] M. Roser, H. Ritchie, E. Ortiz-Ospina, and J. Hasell, 'Coronavirus Pandemic (COVID-19)', Our World in Data, March 04, 2020. https://ourworldindata.org/coronavirus (accessed May 27, 2020).

[9] BBC News, 'Health experts "frustrated" by low UK virus tests', BBC News, April 02, 2020. https://www.bbc.com/news/uk-52130230 (accessed May 07, 2020).

[10] M. C. Bender and A. Restuccia, 'Business Leaders Urge Trump to Dramatically Increase Coronavirus Testing', Wall Street Journal, April 15, 2020. https:/www.wsj.com/articles/business-leaderstell-trump-to-dramatically-increase-coronavirus-testing-11586974130 (accessed May 07, 2020).

[11] GOV.UK, 'Help the government increase coronavirus (COVID-19) testing capacity', GOV.UK, April 10, 2020. https://www.gov.uk/guidance/help-the-government-increase-coronaviruscovid-19-testing-capacity (accessed May 07, 2020).

[12] Washington State Department of Health, 'Testing for COVID-19', Washington State Department of Health, 2020. https://www.doh.wa.gov/Emergencies/NovelCoronavirusOutbreak2020COVID19/TestingforCOVID19 (accessed May 07, 2020).

[13] C. S. Kosack, A.-L. Page, and P. R. Klatser, 'A guide to aid the selection of diagnostic tests', Bulletin of the World Health Organization, vol. 95, no. 9, Art. no. 9, Sep. 2017, doi: 10.2471/BLT.16.187468.

[14] C. Sheridan, 'Fast, portable tests come online to curb coronavirus pandemic', Nat. Biotechnol., Mar. 2020, doi: 10.1038/d41587-020-00010-2.

[15] H. S. Gill, D. D. Denson, B. A. Burris, and M. R. Prausnitz, 'Effect of microneedle design on pain in human volunteers', Clin J Pain, vol. 24, no. 7, Art. no. 7, Sep. 2008, doi: 10.1097/AJP.0b013e31816778f9.

[16] M. I. Haq et al., 'Clinical administration of microneedles: skin puncture, pain and sensation', Biomed Microdevices, vol. 11, Art. no. 1, 2009, doi: 10.1007/s10544-008-9208-1.

[17] S. Kaushik et al., 'Lack of pain associated with microfabricated microneedles', Anesth Analg., vol. 92, no. 2, Art. no. 2, Feb. 2001, doi: 10.1097/00000539-200102000-00041.

[18] G.-S. Liu et al., 'Microneedles for transdermal diagnostics: Recent advances and new horizons', Biomaterials, vol. 232, p. 119740, Feb. 2020, doi: 10.1016/j.biomaterials.2019.119740.

[19] M. T. Arevalo, G. M. Rizzo, R. Polsky, T. Glaros, and P. M. Mach, 'Proteomic Characterization of Immunoglobulin Content in Dermal Interstitial Fluid', J. Proteome Res., vol. 18, no. 6, Art. no. 6, Jun. 2019, doi: 10.1021/acs.jproteome.9b00155.

[20] A. K. Nilsson, U. Sjöbom, K. Christenson, and A. Hellström, 'Lipid profiling of suction blister fluid: comparison of lipids in interstitial fluid and plasma', Lipids Health Dis, vol. 18, Art. no. 1, Dec. 2019, doi: 10.1186/s12944-019-1107-3.

[21] B. Q. Tran et al., 'Proteomic Characterization of Dermal Interstitial Fluid Extracted Using a Novel Microneedle-Assisted Technique', J. Proteome Res., vol. 17, no. 1, Art. no. 1, Jan. 2018, doi: 10.1021/acs.jproteome.7b00642.

[22] J. J. Norman, J. M. Arya, M. A. McClain, P. M. Frew, M. I. Meltzer, and M. R. Prausnitz, 'Microneedle patches: Usability and acceptability for self-vaccination against influenza', Vaccine, vol. 32, no. 16, Art. no. 16, Apr. 2014, doi: 10.1016/j.vaccine.2014.01.076. 
[23] N. G. Rouphael et al., 'The safety, immunogenicity, and acceptability of inactivated influenza vaccine delivered by microneedle patch (TIV-MNP 2015): a randomised, partly blinded, placebo-controlled, phase 1 trial', Lancet, vol. 390, no. 10095, Art. no. 10095, Aug. 2017, doi: 10.1016/S01406736(17)30575-5.

[24] P. Xue, D. C. L. Yeo, Y. J. Chuah, H. L. Tey, Y. Kang, and C. Xu, 'Drug-eluting microneedles for self-administered treatment of keloids', Technology, vol. 02, no. 02, Art. no. 02, Jun. 2014, doi: $10.1142 /$ S2339547814500137.

[25] K. Messacar, S. K. Parker, J. K. Todd, and S. R. Dominguez, 'Implementation of Rapid Molecular Infectious Disease Diagnostics: the Role of Diagnostic and Antimicrobial Stewardship', J. Clin. Microbiol., vol. 55, no. 3, Art. no. 3, Mar. 2017, doi: 10.1128/JCM.02264-16.

[26] A. Niemz, T. M. Ferguson, and D. S. Boyle, 'Point-of-care nucleic acid testing for infectious diseases', Trends Biotechnol, vol. 29, no. 5, Art. no. 5, May 2011, doi: 10.1016/j.tibtech.2011.01.007.

[27] S. Yang and R. E. Rothman, 'PCR-based diagnostics for infectious diseases: uses, limitations, and future applications in acute-care settings', Lancet Infect. Dis., vol. 4, no. 6, Art. no. 6, Jun. 2004, doi: 10.1016/S1473-3099(04)01044-8.

[28] Z. A. Crannell, B. Rohrman, and R. Richards-Kortum, 'Equipment-Free Incubation of Recombinase Polymerase Amplification Reactions Using Body Heat', PLoS ONE, vol. 9, no. 11, Art. no. 11, Nov. 2014, doi: 10.1371/journal.pone.0112146.

[29] M. Fakruddin et al., 'Nucleic acid amplification: Alternative methods of polymerase chain reaction', J Pharm Bioall Sci, vol. 5, no. 4, Art. no. 4, Oct. 2013, doi: 10.4103/0975-7406.120066.

[30] T. Notomi, 'Loop-mediated isothermal amplification of DNA', Nucleic Acids Res., vol. 28, no. 12, Art. no. 12, Jun. 2000, doi: 10.1093/nar/28.12.e63.

[31] Y. Zhao, F. Chen, Q. Li, L. Wang, and C. Fan, 'Isothermal Amplification of Nucleic Acids', Chem. Rev., vol. 115, no. 22, Art. no. 22, Nov. 2015, doi: 10.1021/acs.chemrev.5b00428.

[32] B. Yang, X. Fang, and J. Kong, 'Engineered Microneedles for Interstitial Fluid Cell-Free DNA Capture and Sensing Using Iontophoretic Dual-Extraction Wearable Patch', Adv Funct Mater, vol. n/a, no. n/a, Art. no. n/a, Apr. 2020, doi: 10.1002/adfm.202000591.

[33] B. L. Fernández-Carballo, T. Broger, R. Wyss, N. Banaei, and C. M. Denkinger, 'Toward the Development of a Circulating Free DNA-Based In Vitro Diagnostic Test for Infectious Diseases: A Review of Evidence for Tuberculosis', J Clin Microbiol, vol. 57, no. 4, Art. no. 4, Nov. 2018, doi: 10.1128/JCM.01234-18.

[34] K. G. Weerakoon and D. P. McManus, 'Cell-Free DNA as a Diagnostic Tool for Human Parasitic Infections', Trends Parasitol, vol. 32, no. 5, Art. no. 5, May 2016, doi: 10.1016/j.pt.2016.01.006.

[35] K. W. Ng and S. M. Moghimi, 'Skin Biosensing and Bioanalysis: what the Future Holds', Prec. Nanomed., vol. 1, no. 2, Art. no. 2, Jul. 2018, doi: 10.33218/prnano1(2).180709.1.

[36] G.-S. Liu et al., 'Microneedles for transdermal diagnostics: Recent advances and new horizons', Biomaterials, vol. 232, p. 119740, Feb. 2020, doi: 10.1016/j.biomaterials.2019.119740.

[37] E. V. Mukerjee, S. D. Collins, R. R. Isseroff, and R. L. Smith, 'Microneedle array for transdermal biological fluid extraction and in situ analysis', Sensor Actuat A-Phys, vol. 114, no. 2, Art. no. 2, Sep. 2004, doi: 10.1016/j.sna.2003.11.008.

[38] S. A. Ranamukhaarachchi, C. Padeste, M. Dübner, U. O. Häfeli, B. Stoeber, and V. J. Cadarso, 'Integrated hollow microneedle-optofluidic biosensor for therapeutic drug monitoring in sub-nanoliter volumes', Sci. Rep., vol. 6, no. 1, Art. no. 1, Jul. 2016, doi: 10.1038/srep29075.

[39] L. M. Strambini et al., 'Self-powered microneedle-based biosensors for pain-free high-accuracy measurement of glycaemia in interstitial fluid', Biosens Bioelectron, vol. 66, pp. 162-168, Apr. 2015, doi: 10.1016/j.bios.2014.11.010.

[40] K. Takeuchi, N. Takama, B. Kim, K. Sharma, O. Paul, and P. Ruther, 'Microfluidic chip to interface porous microneedles for ISF collection', Biomed Microdevices, vol. 21, no. 1, Art. no. 1, Mar. 2019, doi: 10.1007/s10544-019-0370-4.

[41] E. Vranić, A. Tucak, M. Sirbubalo, O. Rahić, A. Elezović, and J. Hadžiabdić, 'MicroneedleBased Sensor Systems for Real-Time Continuous Transdermal Monitoring of Analytes in Body Fluids', in CMBEBIH 2019, Cham, 2020, vol. 73, pp. 167-172, doi: 10.1007/978-3-030-17971-7_26. 
[42] P. Xue, L. Zhang, Z. Xu, J. Yan, Z. Gu, and Y. Kang, 'Blood Sampling Using Microneedles as a Minimally Invasive Platform for Biomedical Diagnostics', Appl. Mater. Today, vol. 13, p. 144, Dec. 2018, doi: https://doi.org/10.1016/j.apmt.2018.08.013.

[43] R. L. Smith, S. D. Collins, J. Duy, and T. D. Minogue, 'Silicon microneedle array for minimally invasive human health monitoring', in Microfluidics, BioMEMS, and Medical Microsystems XVI, Feb. 2018, vol. 10491, p. 1049102, doi: 10.1117/12.2299264.

[44] C. Kolluru, M. Williams, J. Chae, and M. R. Prausnitz, 'Recruitment and Collection of Dermal Interstitial Fluid Using a Microneedle Patch', Adv. Healthc. Mater., vol. 8, no. 3, Art. no. 3, Jan. 2019, doi: 10.1002/adhm.201801262.

[45] C. Kolluru, M. Williams, J. S. Yeh, R. K. Noel, J. Knaack, and M. R. Prausnitz, 'Monitoring drug pharmacokinetics and immunologic biomarkers in dermal interstitial fluid using a microneedle patch', Biomed Microdevices, vol. 21, no. 1, Art. no. 1, Feb. 2019, doi: 10.1007/s10544-019-0363-3.

[46] E. Caffarel-Salvador et al., 'Hydrogel-Forming Microneedle Arrays Allow Detection of Drugs and Glucose In Vivo: Potential for Use in Diagnosis and Therapeutic Drug Monitoring', PLOS ONE, vol. 10, no. 12, Art. no. 12, Dec. 2015, doi: 10.1371/journal.pone.0145644.

[47] J. Chen, M. Wang, Y. Ye, Z. Yang, Z. Ruan, and N. Jin, 'Fabrication of sponge-forming microneedle patch for rapidly sampling interstitial fluid for analysis', Biomed Microdevices, vol. 21, no. 3, Art. no. 3, Jul. 2019, doi: 10.1007/s10544-019-0413-x.

[48] R. F. Donnelly et al., 'Hydrogel-Forming Microneedle Arrays for Enhanced Transdermal Drug Delivery', Adv Funct Mater, vol. 22, no. 23, Art. no. 23, Dec. 2012, doi: 10.1002/adfm.201200864.

[49] S. Gholami, M. M. Mohebi, E. Hajizadeh-Saffar, M. H. Ghanian, I. Zarkesh, and H. Baharvand, 'Fabrication of microporous inorganic microneedles by centrifugal casting method for transdermal extraction and delivery', Int. J. Pharm., vol. 558, pp. 299-310, Mar. 2019, doi: 10.1016/j.ijpharm.2018.12.089.

[50] P. Liu et al., 'Polymer microneedles with interconnected porous structures via a phase inversion route for transdermal medical applications', J. Mater. Chem. B, vol. 8, no. 10, Art. no. 10, 2020, doi: 10.1039/C9TB02837D.

[51] T. M. Blicharz et al., 'Microneedle-Based Device for the One-Step Painless Collection of Capillary Blood Samples', Nat. Biomed. Eng., vol. 2, p. 151, Feb. 2018, doi: https://doi.org/10.1038/s41551-018-0194-1.

[52] J. C. Dale and S. G. Ruby, 'Specimen collection volumes for laboratory tests', Arch. Pathol. Lab. Med., vol. 127, no. 2, Art. no. 2, Feb. 2003, doi: 10.1043/00039985(2003)127<162:SCVFL >2.0.CO;2.

[53] H. H. Kessler, Molecular Diagnostics of Infectious Diseases, 3rd ed. Walter de Gruyter, 2014.

[54] F. Poitout-Belissent, A. Aulbach, N. Tripathi, and L. Ramaiah, 'Reducing blood volume requirements for clinical pathology testing in toxicologic studies - points to consider', Vet Clin Path, vol. 45, no. 4, Art. no. 4, 2016, doi: 10.1111/vcp.12429.

[55] R. S. Sista et al., 'Digital Microfluidic Platform to Maximise Diagnostic Tests with Low Sample Volumes from Newborns and Pediatric Patients', Diagnostics (Basel), vol. 10, no. 1, Art. no. 1, Jan. 2020, doi: 10.3390/diagnostics 10010021 .

[56] World Health Organization, 'Laboratory-based evaluation of 19 commercially available rapid diagnostic tests for tuberculosis', 2, Oct. 2008. doi: 10.2471/TDR.08.9789241597111.

[57] World Health Organization, N. Dhingra, and Safe Injection Global Network, WHO guidelines on drawing blood: best practices in phlebotomy. 2010.

[58] R. M. Taylor, P. R. Miller, P. Ebrahimi, R. Polsky, and J. T. Baca, 'Minimally-invasive, microneedle-array extraction of interstitial fluid for comprehensive biomedical applications: transcriptomics, proteomics, metabolomics, exosome research, and biomarker identification', Lab. Anim., vol. 52, no. 5, Art. no. 5, Oct. 2018, doi: 10.1177/0023677218758801.

[59] C. G. Li, H.-A. Joung, H. Noh, M.-B. Song, M.-G. Kim, and H. Jung, 'One-touch-activated blood multidiagnostic system using a minimally invasive hollow microneedle integrated with a paperbased sensor', Lab Chip, vol. 15, no. 16, Art. no. 16, Aug. 2015, doi: 10.1039/c5lc00669d. 
[60] D. Nicholas et al., 'Rapid paper based colorimetric detection of glucose using a hollow microneedle device', Int. J. Pharm., vol. 547, no. 1-2, Art. no. 1-2, Aug. 2018, doi: 10.1016/j.ijpharm.2018.06.002.

[61] J. W. Coffey, S. R. Corrie, and M. A. F. Kendall, 'Early circulating biomarker detection using a wearable microprojection array skin patch', Biomaterials, vol. 34, no. 37, Art. no. 37, Dec. 2013, doi: 10.1016/j.biomaterials.2013.08.078.

[62] S. R. Corrie, G. J. P. Fernando, M. L. Crichton, M. E. G. Brunck, C. D. Anderson, and M. A. F. Kendall, 'Surface-modified microprojection arrays for intradermal biomarker capture, with low nonspecific protein binding', Lab Chip, vol. 10, no. 20, Art. no. 20, Oct. 2010, doi: 10.1039/c01c00068j.

[63] K. T. Lee et al., 'Capture of the Circulating Plasmodium falciparum Biomarker HRP2 in a Multiplexed Format, via a Wearable Skin Patch', Anal. Chem., vol. 86, no. 20, Art. no. 20, Oct. 2014, doi: $10.1021 /$ ac5031682.

[64] D. A. Muller, S. R. Corrie, J. Coffey, P. R. Young, and M. A. Kendall, 'Surface modified microprojection arrays for the selective extraction of the dengue virus NS1 protein as a marker for disease', Anal. Chem., vol. 84, no. 7, Art. no. 7, Apr. 2012, doi: 10.1021/ac2034387.

[65] K. W. Ng, W. M. Lau, and A. C. Williams, 'Towards pain-free diagnosis of skin diseases through multiplexed microneedles: biomarker extraction and detection using a highly sensitive blotting method', Drug Deliv Transl Re, vol. 5, no. 4, Art. no. 4, 2015, doi: 10.1007/s13346-015-0231-5.

[66] S. Totti, K. W. Ng, L. Dale, G. Lian, T. Chen, and E. G. Velliou, 'A novel versatile animal-free 3D tool for rapid low-cost assessment of immunodiagnostic microneedles', Sensor Actuat B-Chem, vol. 296, p. 126652, Oct. 2019, doi: 10.1016/j.snb.2019.126652.

[67] J. W. Coffey, S. R. Corrie, and M. A. F. Kendall, 'Rapid and Selective Sampling of IgG from Skin In Less Than 1 min Using a High Surface Area Wearable Immunoassay Patch', Biomaterials, vol. 170, pp. 49-57, Jul. 2018, doi: https://doi.org/10.1016/j.biomaterials.2018.03.039.

[68] D. Al Sulaiman et al., 'Hydrogel-Coated Microneedle Arrays for Minimally Invasive Sampling and Sensing of Specific Circulating Nucleic Acids from Skin Interstitial Fluid', ACS Nano, vol. 13, no. 8, Art. no. 8, Aug. 2019, doi: 10.1021/acsnano.9b04783.

[69] Z. Li and G.-Y. Chen, 'Current Conjugation Methods for Immunosensors', Nanomaterials, vol. 8, no. 5, Art. no. 5, Apr. 2018, doi: 10.3390/nano8050278.

[70] P. Bollella, S. Sharma, A. E. G. Cass, and R. Antiochia, 'Minimally-invasive Microneedlebased Biosensor Array for Simultaneous Lactate and Glucose Monitoring in Artificial Interstitial Fluid', Electroanalysis, vol. 31, no. 2, Art. no. 2, Jan. 2019, doi: https://doi.org/10.1002/elan.201800630.

[71] J. Gao, W. Huang, Z. Chen, C. Yi, and L. Jiang, 'Simultaneous detection of glucose, uric acid and cholesterol using flexible microneedle electrode array-based biosensor and multi-channel portable electrochemical analyser', Sensor Actuat B-Chem, vol. 287, pp. 102-110, May 2019, doi: 10.1016/j.snb.2019.02.020.

[72] D. H. Keum et al., 'Microneedle Biosensor for Real-Time Electrical Detection of Nitric Oxide for In Situ Cancer Diagnosis During Endomicroscopy', Advanced Healthcare Materials, p. n/a-n/a, Feb. 2015, doi: 10.1002/adhm.201500012.

[73] E. Skaria, B. A. Patel, M. S. Flint, and K. W. Ng, 'Poly(lactic acid)/Carbon Nanotube Composite Microneedle Arrays for Dermal Biosensing', Anal. Chem., vol. 91, no. 7, Art. no. 7, Apr. 2019, doi: 10.1021/acs.analchem.8b04980.

[74] W. H. Smart and K. Subramanian, 'The use of silicon microfabrication technology in painless blood glucose monitoring', Diabetes Technol The, vol. 2, no. 4, Art. no. 4, 2000, doi: 10.1089/15209150050501961.

[75] S. Babity, M. Roohnikan, and D. Brambilla, 'Advances in the Design of Transdermal Microneedles for Diagnostic and Monitoring Applications', Small, vol. 14, no. 49, Art. no. 49, Oct. 2018, doi: 10.1002/smll.201803186.

[76] A. V. Ganesan, 'A Novel MEMS Based Immunosensor for Ebola Virus Detection', in Volume 7B: Fluids Engineering Systems and Technologies, San Diego, California, USA, Nov. 2013, p. V07BT08A074, doi: 10.1115/IMECE2013-66025. 
[77] A. V. Ganesan, D. Kishore Kumar, A. Banerjee, and S. Swaminathan, 'MEMS based microfluidic system for HIV detection', in 2013 13th IEEE International Conference on Nanotechnology (IEEE-NANO 2013), Beijing, China, Aug. 2013, pp. 557-560, doi: 10.1109/NANO.2013.6721005.

[78] Global Preparedness Monitoring Board, 'A World at Risk: Annual Report on Global Preparednessfor Health Emergencies', Sep. 2019. https://apps.who.int/gpmb/assets/annual_report/GPMB_annualreport_2019.pdf (accessed May 07, 2020).

[79] F. van Mierlo, R. Jonczyk, and V. Qian, 'Next generation Direct Wafer® technology delivers low cost, high performance to silicon wafer industry', Energy Procedia, vol. 130, pp. 2-6, Sep. 2017, doi: 10.1016/j.egypro.2017.09.403.

[80] H. R. Nejad, A. Sadeqi, G. Kiaee, and S. Sonkusale, 'Low-cost and cleanroom-free fabrication of microneedles', MICROSYST NANOENG, vol. 4, no. 1, Art. no. 1, Jan. 2018, doi: 10.1038/micronano.2017.73.

[81] G. L. Hortin, 'Does Point-Of-Care Testing Save Money or Cost More?', Lab Med, vol. 36, no. 8, pp. 465-467, Aug. 2005, doi: 10.1309/AJ9D0GHYYWEDYHPD.

[82] A. St John and C. P. Price, 'Economic Evidence and Point-of-Care Testing', Clin Biochem Rev, vol. 34, no. 2, pp. 61-74, Aug. 2013, Accessed: June 30, 2020. [Online]. Available: https://www.ncbi.nlm.nih.gov/pmc/articles/PMC3799220/.

[83] E. Larrañeta, R. E. M. Lutton, A. D. Woolfson, and R. F. Donnelly, 'Microneedle arrays as transdermal and intradermal drug delivery systems: Materials science, manufacture and commercial development', Mat Sci Eng R, vol. 104, pp. 1-32, Jun. 2016, doi: 10.1016/j.mser.2016.03.001. 